\title{
Working beyond your job plan
}

CLINICAL REFLECTION

\section{Alex Till \& Jan Wise}

\begin{abstract}
SUMMARY
A job plan is part of a modern contract, informing employee and employer what they are paid to do. It is not a mandatory, inflexible tablet of stone, but frequently National Health Service (NHS) clinicians find themselves working, to their detriment, in excess of what they have agreed to do, by bridging the gap between resources and demand. So what do we do when we are persistently going above and beyond and there is no more left to give: how can we fix the system, and who really gets left behind?
\end{abstract}

\section{DECLARATION OF INTEREST}

None

In today's economic climate, and an increasingly fragmented system, as NHS clinicians we frequently find ourselves reflecting on the quality and quantity of care that we can deliver. There is growing demand and constrained resources. Colleagues are struggling under the emotional burden of failing to provide the quality of service that was once promised and of matching what society increasingly wants (and deserves) against what many argue that, in reality, they need.

Out of compassion for our patients, determination to succeed in our careers, drive to maintain our professionalism and, unfortunately, often a fear about how this might be questioned by our employer and regulatory body should we fail, we regularly go that extra mile. Skipping breaks, staying late, driving home exhausted, and working at weekends to deliver care and meet the growing bureaucratic burden of clinical administration, training, appraisal and revalidation: at what point do we halt? At what point do we question whether we are pushing ourselves too far?

The tension between wanting to do a good job and what it takes to do it can often seem insurmountable, for the level of attention and steps necessary to match resources with demand, attain our professional aspirations and meet our statutory requirements do not fit into the time available to us. They quickly become 'time stealers', eroding the time we have to deliver patient care and eating into our personal lives.

This cannot continue, but how do we deal with it and are we ourselves partly to blame?

\section{Working with, rather than fighting against, our non-clinical colleagues}

One step removed from patient contact, but equally under pressure, our colleagues in management, commissioning and policy face a challenging task; we should work with them, rather than fight against them. Not directly on the front line, they struggle more to feel, see or hear for themselves the effects that this pressure has on patients; and without firm evidence highlighting how strained resources are resulting in falling standards and clinicians under pressure, all they see is shroudwaving over the impact of spending cuts and all they hear is the bleating of a frightened flock. They too work under the constraints of the system and, without evidence, they have no recourse to call for support and are obliged to accept, if not believe, that the status quo is working. Ultimately, this stretches the service further to meet key performance indicators and inadvertently takes advantage of clinicians' generosity.

Although we, as a profession, are always here when people need us, it is not someone else's, but our responsibility to present the evidence for the time it takes to complete tasks safely, refuse unfunded activity and prioritise our continuing professional development needs. We need to engage in working collaboratively: teaching, shadowing and mentoring one another. Without this, there will be no change in service, or budget, as we are seen to be coping.

\section{Protected time is not protected enough}

Previous attempts to overcome time pressures by 'supporting professional activities' (SPAs) time for consultants (NHS Employers 2013) - recommended by the Royal College of Psychiatrists (2017) to be set at 1.5 SPAs per week for revalidation and appraisal - appear to be insufficient; time and again we see both senior and junior colleagues struggling. At what point do we accept tasks as being mandatory, rather than 'extra-curricular' and to be done in our own time?

Consultants, sometimes begging for more time, are told they are capped at 10 programmed activities (PAs) per week. However, although stated in the current consultant contract (NHS Employers 2013), there is no statutory basis for this, and job
Alex Till is a psychiatric trainee and a member of the Royal College of Psychiatrists' Psychiatric Trainees' Committee, a Leadership and Management Fellow with Mersey Care NHS Foundation Trust, and a National Medical Director's Clinical Fellow (2016/2017 cohort) at the Faculty of Medical Leadership and Management and NHS England. Jan Wise is a consultant psychiatrist with Central and North West London NHS Foundation Trust. She chairs and sits on several BMA committees involved in trade union duties, caring for doctors so that they can care for patients. Correspondence Dr Alex Till, Mersey Care NHS Foundation Trust, Trust Headquarters, V7 Building, Kings Business Park, Prescot, Liverpool L34 1PJ, UK. Email: alextill54@gmail.com

\section{Copyright and usage} (C) The Royal College of Psychiatrists 2017 
plans above 10 PAs widely exist throughout the UK. For our trainees, who are still learning their craft, facing pressures of revising for exams and meeting their wider training needs, their protected training time is increasingly eroded by a clinical environment pressuring them into front-line service delivery.

However, is there hope around the corner in the new consultant contract (currently being negotiated) and exception reporting in the new junior doctor contract (NHS Employers 2017), or will these simply create yet further pressures on an already strained workforce?

\section{A new clinical environment}

Contracts aside, we must realise that the provision of care has moved on. In modern medicine, with a wide variety of multidisciplinary healthcare professionals adopting a team-based approach, a single clinician is just one of many participants in a system very different from the traditional one-toone therapeutic relationship historically observed between patient and clinician.

However, psychologically, have we kept up with this and moved on as a profession? At what point do we shift the balance from upholding our professional duty to recognising that in managed healthcare systems, with all their complexities and interactions, we are not responsible for the whole patient journey? Perhaps too many of us are caught up with a feeling of personal responsibility for the welfare of those who suffer, or are trying to retain a perceived last sense of professional autonomy as we see our system crumbling around us, rather than embracing the concept of team, organisation and system accountability. When did our profession, which claims expertise in establishing and maintaining boundaries, develop such porous ones?

\section{Speaking up for change}

The danger is that we mask the problem, bridge this gap and personally bear the psychological weight of these issues. Unless clinicians engage, stand up and say 'we are all in this together', society will not have the opportunity to join the conversation over the degree of funding for services and whether they are the services they want; our regulators will not move towards embracing a just culture (Dekker 2016) with a greater understanding of human error and systems failures, and we, as clinicians, will continue to bear the burden.
We already have a recruitment crisis in both the NHS in general and psychiatry in particular, and unless we address these issues and act as agents for change, we will eventually fail to carry this weight. We will have a larger number of clinicians suffering 'burnout', taking long-term medical absence from work or retiring early. Not only will clinicians themselves suffer, but ultimately so will the system and our patients.

Our very act of caring and of wishing to do the best for our patients has made it difficult to present the evidence. We have compensated for a system where supply does not match demand. But the abyss is approaching and we must question whether our good will and humanity have, in part, contributed to this potentially catastrophic situation.

\section{A shift in psychology}

We need to modify our behaviour, make a psychological shift, and question whether we should continually go the extra mile to bridge the gap and sustain the view that our system is working. Clinicians should not suffer individually. It is now, more than ever, necessary to evidence front-line pressures, highlight the time it takes to do a task and decline to carry out tasks for which no time has been allocated and that are not a clinical emergency. We have a contract that allows us to decline non-emergency work in premium time; we should use it (Wannan 2015).

When the taskmaster, whether punitive superego or external force beyond our control, says 'work longer, work harder', we must think about the long-term consequences and ask ourselves the following questions: Are you being fair to yourself? How sustainable is this? Is it really a one-off? If the answer to any of these questions is no, then we must stand up and make our voices heard, not only for ourselves as individuals and clinicians, but for the NHS and our patients.

\section{References}

Dekker S (2016) Just Culture: Restoring Trust and Accountability in Your Organization (3rd edn). CRC Press.

NHS Employers (2013) Terms and Conditions - Consultants (England) 2003 (version 9). NHS Employers.

NHS Employers (2017) Terms and Conditions of Service for NHS Doctors and Dentists in Training (England) 2016 (Version 2). NHS Employers.

Royal College of Psychiatrists (2017) Safe Patients and High-Quality Services: Job Descriptions for Consultant Psychiatrists (College Report CR207). Royal College of Psychiatrists (in press).

Wannan G, Wise MEJ (2015) Are psychiatrists only fools and horses to be open all hours? BJPsych Bulletin, 39: 265-7. 